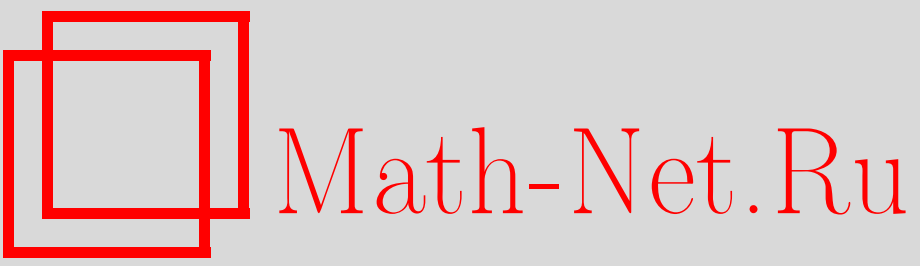

В. В. Жаринов, Когомологии Хохшильда алгебры гладких функций на торе, TMФ, 2005, том 144, номер 3, 435-452

DOI: https://doi.org/10.4213/tmf1869

Использование Общероссийского математического портала Math-Net.Ru подразумевает, что вы прочитали и согласны с пользовательским соглашением

http://www.mathnet.ru/rus/agreement

Параметры загрузки:

IP : 54.205 .225 .156

26 апреля 2023 г., 11:26:03 
ТЕОРЕТИЧЕСКАЯ

И МАТЕМАТИЧЕСКАЯ

ФИЗИКА

Том 144, № 3

сентябрь, 2005

(C) 2005 г.

В.В. Жаринов*

\section{КОГОМОЛОГИИ ХОХШИЛЬДА АЛГЕБРЫ ГЛАДКИХ ФУНКЦИЙ НА ТОРЕ}

Вычислены когомологии Хохшильда алгебры гладких функций на конечномерном вещественном торе с коэффициентами в присоединенном представлении, для чего развитая ранее техника модифицирована на дискретный случай.

Ключевые слова: когомологии Хохшильда, присоединенное представление, комплекс де Рама на целочисленной решетке.

В предыдушей работе [1] аналитическими методами вычислены когомологии Хохшильда алгебры гладких функций на конечномерном вешественном пространстве с коэффициентами в присоединенном представлении. В предлагаемой работе вычислены когомологии Хохшильда алгебры гладких функций на конечномерном вешественном торе с коэффициентами в присоединенном представлении (теорема 5.2), для чего развитая в [2], [3] и [1] техника модифицирована на дискретный случай. Чтобы продемонстрировать преемственность метода вычислений, используется схема, предложенная в [1], а ради замкнутости изложения ряд определений и утверждений оттуда повторены. В дополнении излагаются элементы когомологий де Рама на целочисленной решетке.

\section{1. КОГОМОЛОГИИ ХОХШИЛЬДА}

Пусть заданы $\mathbb{F}$ - поле характеристики нуль, $\mathfrak{A}$ - ассоциативная алгебра над $\mathbb{F}, \mathfrak{B}$ бимодуль над $\mathfrak{A}$.

Пусть $\otimes \mathfrak{A}=\bigoplus_{q \geqslant 0} \otimes^{q} \mathfrak{A}$ - тензорная алгебра линейного пространства $\mathfrak{A}, \mathcal{C}(\mathfrak{A} ; \mathfrak{B})=$ $\bigoplus_{q \geqslant 0} \mathcal{C}^{q}(\mathfrak{A} ; \mathfrak{B})$ - линейное пространство всех коцепей на $\mathfrak{A}$ со значениями в $\mathfrak{B}$, где $\mathcal{C}^{q}(\mathfrak{A} ; \mathfrak{B})=\operatorname{Hom}_{\mathbb{F}}(\otimes q \mathfrak{A} ; \mathfrak{B})$. Кограничный оператор $\delta$ на $\mathcal{C}(\mathfrak{A} ; \mathfrak{B})$ задается правилом

$$
\begin{aligned}
& \delta \omega\left(a_{1}, \ldots, a_{q+1}\right)=a_{1} \omega\left(a_{2}, \ldots, a_{q+1}\right)+ \\
& \quad+\sum_{1 \leqslant r \leqslant q}(-1)^{r} \omega\left(a_{1}, \ldots, a_{r} a_{r+1}, \ldots, a_{q+1}\right)+(-1)^{q+1} \omega\left(a_{1}, \ldots, a_{q}\right) a_{q+1},
\end{aligned}
$$

* Математический институт им. В. А. Стеклова РАН, Москва, Россия. E-mail: zharinov@mi.ras.ru 
где $q=0,1, \ldots, \omega \in \mathcal{C}^{q}(\mathfrak{A} ; \mathfrak{B}), a_{1}, \ldots, a_{q+1} \in \mathfrak{A}, \delta \omega \in \mathcal{C}^{q+1}(\mathfrak{A} ; \mathfrak{B})$. Легко проверяется, что $\delta \circ \delta=0$ (здесь и всюду ниже значок о означает композицию отображений), так что определен комплекс $\{\mathcal{C}(\mathfrak{A} ; \mathfrak{B}) ; \delta\}$ с когомологиями

$$
H\{\mathcal{C}(\mathfrak{A} ; \mathfrak{B}) ; \delta\}=H(\mathfrak{A} ; \mathfrak{B})=\bigoplus_{q \geqslant 0} H^{q}(\mathfrak{A} ; \mathfrak{B}) .
$$

Над всякой ассоциативной алгеброй $\mathfrak{A}$ определены два естественных бимодуля: тривиальныцй, здесь $\mathfrak{B}=\mathbb{F}$, действия $a x=x a=0$ для всех $a \in \mathfrak{A}, x \in \mathbb{F}$;

присоединенныц й, здесь $\mathfrak{B}=\mathfrak{A}$, действия $a b, b a$ суть произведения в алгебре $\mathfrak{A}$ для всех $a \in \mathfrak{A}, b \in \mathfrak{B}=\mathfrak{A}$.

ПРЕДЛОЖенИЕ 1.1. Если алгебра $\mathfrak{A}$ унитальная (т.е. содержит единичный элемент), то когомологии

$$
H^{q}(\mathfrak{A} ; \mathbb{F})= \begin{cases}\mathbb{F}, & q=0 \\ 0, & q=1,2, \ldots\end{cases}
$$

\section{2. АЛГЕБРА ГЛАДКИХ ФУНКЦИЙ НА ТОРЕ}

Начиная с этого момента $\mathbb{F}=\mathbb{C}, \mathbb{Z}=\{0, \pm 1, \pm 2, \ldots\}$ - кольцо целых чисел, $\mathbb{T}=$ $\mathbb{R}^{D} / 2 \pi \mathbb{Z}^{D}$ - вешественный $D$-мерный тор, $\mathbb{L}=\mathbb{Z}^{D}$ - его решетка периодов, $\mathfrak{A}=\mathcal{E}(\mathbb{T})=$ $\mathcal{C}^{\infty}(\mathbb{T} ; \mathbb{C})$ - алгебра всех гладких комплексных функций на $\mathbb{T}$ с естественной топологией равномерной сходимости вместе с производными всех порядков.

В случае тривиальных коэффициентов пространства $\mathcal{C}^{q}(\mathcal{E}(\mathbb{T}) ; \mathbb{C})=\mathcal{E}\left(\mathbb{T}^{q}\right)^{\prime}-$ линейные пространства, дуальные к пространствам $\mathcal{E}\left(\mathbb{T}^{q}\right)$, наделенные сильной топологией, так что $q$-коцепи суть обобщенные функции на $\mathbb{T}^{q}=\times^{q} \mathbb{T}$. Когомологии комплекса $\{\mathcal{C}(\mathcal{E}(\mathbb{T}) ; \mathbb{C}) ; \delta\}$ описьваются предложением 1.1, поскольку алгебра $\mathcal{E}(\mathbb{T})$ содержит единичньй элемент - функцию $e(x) \equiv 1$.

Остальная часть данной статьи посвящена когомологиям алгебры $\mathcal{E}(\mathbb{T})$ с коэффициентами в присоединенном бимодуле $\mathfrak{B}=\mathcal{E}(\mathbb{T})$. Здесь пространства $\mathcal{C}^{q}(\mathcal{E}(\mathbb{T}) ; \mathcal{E}(\mathbb{T}))$ линейные пространства всех непрерывных $q$-линейных отображений из $\mathcal{E}(\mathbb{T})^{q}$ в $\mathcal{E}(\mathbb{T})$, наделенные сильной топологией. Справедливо представление $\mathcal{C}^{q}(\mathcal{E}(\mathbb{T}) ; \mathcal{E}(\mathbb{T}))=$ $\mathcal{E}(\mathbb{T}) \widehat{\otimes} \mathcal{E}\left(\mathbb{T}^{q}\right)^{\prime}$, где значок означает замыкание тензорного произведения в подходяшей топологии, которая для нас сейчас не важна [4]. В частности, $q$-коцепи суть $\mathcal{E}(\mathbb{T})$-значные обобщенные функции на $\mathbb{T}^{q}$.

\section{3. ПРЕОБРАЗОВАНИЕ ФУРЬЕ}

Алгебра $\mathcal{E}(\mathbb{T})$ всех гладких функций на торе $\mathbb{T}$ отождествляется с алгеброй всех гладких функций на вещественном пространстве $\mathbb{R}^{D}, 2 \pi$-периодических по каждому аргументу. Для $m=\left(\mu^{1}, \ldots, \mu^{D}\right) \in \mathbb{L}$ и $x=\left(x_{1}, \ldots, x_{D}\right) \in \mathbb{R}^{D}$ положим

$$
(m, x)=\sum_{1 \leqslant \alpha \leqslant D} \mu^{\alpha} x_{\alpha}
$$


каждому $m \in \mathbb{L}$ поставим в соответствие функцию

$$
e^{i m} \in \mathcal{E}(\mathbb{T}), \quad \text { где } e^{i m}(x)=e^{i(m, x)} \text { для всех } \quad x \in \mathbb{R}^{D} .
$$

Пусть $\Phi^{q}, q=0,1, \ldots$, суть линейные пространства всех медленно растущих функций на $\mathbb{L}^{q}$, т.е. всех функций $f=f\left(m_{1}, \ldots, m_{q}\right), m_{1}, \ldots, m_{q} \in \mathbb{L}$, растущих не быстрее некоторой степени $|\mathbf{m}|$ при $|\mathbf{m}|=\left|m_{1}\right|+\cdots+\left|m_{q}\right| \rightarrow \infty$, где $\left|m_{k}\right|=\left|\mu_{k}^{1}\right|+\cdots+\left|\mu_{k}^{D}\right|$ для всех $m_{k}=\left(\mu_{k}^{1}, \ldots, \mu_{k}^{D}\right) \in \mathbb{L}, 1 \leqslant k \leqslant q$. В частности, $\Phi^{0}=\mathbb{C}$.

Преобразование $\Phi$ урье $\omega \mapsto F \omega$, действуюшее по формуле

$$
F \omega\left(m_{1}, \ldots, m_{q}\right)=\omega\left(e^{i m_{1}}, \ldots, e^{i m_{q}}\right), \quad \omega \in \mathcal{E}\left(T^{q}\right)^{\prime}, \quad m_{1}, \ldots, m_{q} \in \mathbb{L},
$$

есть изоморфизм $F: \mathcal{E}\left(\mathbb{T}^{q}\right)^{\prime} \simeq \Phi^{q}$ линейных пространств, этот изоморфизм становится топологическим при наделении линейных пространств $\mathcal{E}\left(\mathbb{T}^{q}\right)^{\prime}$ и $\Phi^{q}$ естественными топологиями [5]. Отождествив $F \equiv \mathrm{id}_{\mathcal{E}(\mathbb{T})} \otimes F$, распространим преобразование Фурье на $\mathcal{E}(\mathbb{T}) \widehat{\otimes} \mathcal{E}\left(\mathbb{T}^{q}\right)^{\prime}$ и придем к изоморфизму

$$
F: \mathcal{C}^{q}(\mathcal{E}(\mathbb{T}) ; \mathcal{E}(\mathbb{T})) \simeq \mathfrak{F}^{q}=\mathcal{E}(\mathbb{T}) \widehat{\otimes} \Phi^{q}
$$

“Подкрученное" преобразование $\widetilde{F}: \mathcal{C} q(\mathcal{E}(\mathbb{T}) ; \mathcal{E}(\mathbb{T})) \simeq \mathfrak{F}^{q}$ задается правилом

$$
\widetilde{F} \omega\left(x ; \xi_{1}, \ldots, \xi_{q}\right)=\exp \left\{-i \sum_{1 \leqslant k \leqslant q}\left(m_{k}, x\right)\right\} \omega\left(x ; e^{i m_{1}}, \ldots, e^{i m_{q}}\right)
$$

для всех $\omega \in \mathcal{C}^{q}(\mathcal{E}(\mathbb{T}) ; \mathcal{E}(\mathbb{T})), x \in \mathbb{R}^{D}, m_{1}, \ldots, m_{q} \in \mathbb{L}$, и определяет изоморфизм

$$
\widetilde{F}: \mathcal{C}(\mathcal{E}(\mathbb{T}) ; \mathcal{E}(\mathbb{T})) \simeq \mathfrak{F}=\bigoplus_{q \geqslant 0} \mathfrak{F}^{q}
$$

Кограничный оператор $\delta: \mathcal{C}(\mathcal{E}(\mathbb{T}) ; \mathcal{E}(\mathbb{T})) \rightarrow \mathcal{C}(\mathcal{E}(\mathbb{T}) ; \mathcal{E}(\mathbb{T}))$ индуцирует дифференциал $d_{\mathfrak{F}}=\widetilde{F} \circ \delta \circ \widetilde{F}^{-1}: \mathfrak{F} \rightarrow \mathfrak{F}$, действуюший по правилу

$$
\begin{aligned}
& d_{\mathfrak{F}} f\left(x ; m_{1}, \ldots, m_{q+1}\right)=f\left(x ; m_{2}, \ldots, m_{q+1}\right)+ \\
& \quad+\sum_{1 \leqslant k \leqslant q}(-1)^{k} f\left(x ; m_{1}, \ldots, m_{k}+m_{k+1}, \ldots, m_{q+1}\right)+(-1)^{q+1} f\left(x ; m_{1}, \ldots, m_{q}\right)
\end{aligned}
$$

для всех $f \in \mathfrak{F}^{q}, x \in \mathbb{T}, m_{1}, \ldots, m_{q+1} \in \mathbb{L}$.

Итак, определены комплекс $\left\{\mathfrak{F} ; d_{\mathfrak{F}}\right\}$ и изоморфизмы комплексов $\widetilde{F}$ : $\{\mathcal{C}(\mathcal{E}(\mathbb{T}) ; \mathcal{E}(\mathbb{T})) ; \delta\} \simeq\left\{\mathfrak{F} ; d_{\mathfrak{F}}\right\} \quad$ и соответствуюших пространств когомологий $\widetilde{F}:$ $H(\mathcal{E}(\mathbb{T}) ; \mathcal{E}(\mathbb{T})) \simeq H(\mathfrak{F}) . \quad$ Очевидно, дифференциал $d_{\mathfrak{F}}=\operatorname{id}_{\mathcal{E}(\mathbb{T})} \otimes d$, где дифференциал $d: \Phi \rightarrow \Phi, \quad \Phi=\bigoplus_{q \geqslant 0} \Phi^{q}$, действует по "суженному" правилу (его явный вид приведен ниже). Таким образом, определен вспомогательный комплекс $\{\Phi ; d\}$ с пространством когомологий $H(\Phi)$ и справедливо

ПРЕДЛОЖЕНИЕ 3.1. Имеет место изоморфизм

$$
H(\mathcal{E}(\mathbb{T}) ; \mathcal{E}(\mathbb{T})) \simeq \mathcal{E}(\mathbb{T}) \widehat{\otimes} H(\Phi)
$$




\section{4. ЛИНЕЙНЫЕ ОПЕРАЦИИ НА Ф}

\section{1. Мультииндексные обозначения:}

$\mathbb{Z}=\{0, \pm 1, \pm 2, \ldots\} \supset \mathbb{Z}_{+}=\{0,1,2, \ldots\} \supset \mathbb{N}=\{1,2, \ldots\} ;$

$[\mu, \nu]=\{\mu, \mu+1, \mu+2, \ldots, \nu\} \subset \mathbb{Z}$ для любых $\mu, \nu \in \mathbb{Z}, \mu \leqslant \nu ;$

$\mathbb{L}=\mathbb{Z}^{D}=\left\{m=\left(\mu^{1}, \ldots, \mu^{D}\right) ; \mu^{\alpha} \in \mathbb{Z}, \alpha \in[1, D]\right\}, \mathbb{L}_{+}=\mathbb{Z}_{+}^{D}$, где $D$ - размерность тора $\mathbb{T}=\mathbb{R}^{D} / 2 \pi \mathbb{Z}^{D}$

$|m|=\left|\mu^{1}\right|+\cdots+\left|\mu^{D}\right|$ для каждого $m \in \mathbb{L}$;

$e_{\alpha}=(0, \ldots, 1, \ldots, 0) \in \mathbb{L}$, где 1 стоит на $\alpha$-м месте, $\alpha \in[1, D]$;

$[1, D]_{q}=\left\{\mathrm{A}=\left(\alpha_{1}, \ldots, \alpha_{q}\right) ; \alpha_{i} \in[1, D], i \in[1, q]\right\}, q \in \mathbb{N}$

$|\mathrm{A}|=q$ для каждого $\mathrm{A} \in[1, D]_{q}$;

$\mathrm{A}^{\prime}=\left(\alpha_{q}, \ldots, \alpha_{1}\right), \quad \alpha \mathrm{A}=\left(\alpha, \alpha_{1}, \ldots, \alpha_{q}\right), \quad \mathrm{A}^{i}=\left(\alpha_{1}, \ldots, \check{\alpha}_{i}, \ldots, \alpha_{q}\right)$, для всех $\mathrm{A}=$ $\left(\alpha_{1}, \ldots, \alpha_{q}\right), \alpha \in[1, D], i \in[1, q]$, значок $`$ означает, что соответствуюший индекс пропушен;

$$
\mathbb{V}=\mathbb{C}^{D}=\mathbb{C} \otimes_{\mathbb{Z}} \mathbb{L}
$$

4.2. Операции. Нам понадобятся следующие функции из $\Phi$ :

$1_{q} \in \Phi^{q}$, где $1_{q}\left(m_{1}, \ldots, m_{q}\right)=1$ для всех $m_{1}, \ldots, m_{q} \in \mathbb{L}, q \in \mathbb{N}$;

$\xi^{\alpha} \in \Phi^{1}$, где $\xi^{\alpha}(m)=\mu^{\alpha}$ для всех $m=\left(\mu^{1}, \ldots, \mu^{D}\right) \in \mathbb{L}, \alpha \in[1, D]$.

На линейном пространстве $\Phi$ определена структура ассоциативной алгебры правилом $f \in \Phi^{p}, g \in \Phi^{q} \mapsto f \otimes g \in \Phi^{p+q}$, где

$$
f \otimes g\left(m_{1}, \ldots, m_{p+q}\right)=f\left(m_{1}, \ldots, m_{p}\right) g\left(m_{p+1}, \ldots, m_{p+q}\right)
$$

для всех $m_{1}, \ldots, m_{p+q} \in \mathbb{L}, p, q \in \mathbb{Z}_{+}$.

Кроме того, на $\Phi$ определена основная операция - дифференциал $d$, действуюший по правилу

$$
\begin{aligned}
& d f\left(m_{1}, \ldots, m_{q+1}\right)=f\left(m_{2}, \ldots, m_{q+1}\right)+ \\
& \quad+\sum_{1 \leqslant r \leqslant q}(-1)^{r} f\left(m_{1}, \ldots, m_{r}+m_{r+1}, \ldots, m_{q+1}\right)+(-1)^{q+1} f\left(m_{1}, \ldots, m_{q}\right)
\end{aligned}
$$

для всех $f \in \Phi^{q}, m_{1}, \ldots, m_{q+1} \in \mathbb{L}, q \in \mathbb{Z}_{+}$.

Легко проверяется

ПРЕДЛОЖенИЕ 4.1. Отображение $d: \Phi \rightarrow \Phi$ является косым дифферениированием алгебры $\Phi$, т.е.

$$
d(f \otimes g)=(d f) \otimes g+(-1)^{p} f \otimes(d g)
$$

для всех $f \in \Phi^{p}, \quad g \in \Phi^{q}, \quad p, q \in \mathbb{Z}_{+}$.

Введем следующие линейные отображения на $\Phi$.

Линейное отображение $\varkappa: \Phi \rightarrow \Phi$ зададим правилом

$$
\varkappa f\left(m_{1}, \ldots, m_{q-1}\right)=f\left(0, m_{1}, \ldots, m_{q-1}\right)
$$


для всех $f \in \Phi^{q}, m_{1}, \ldots, m_{q-1} \in \mathbb{L}, q \in \mathbb{Z}_{+}$. В частности, $\varkappa\left(\Phi^{0}\right)=0$.

Линейное отображение $\lambda=1_{1} \otimes: \Phi \rightarrow \Phi$ зададим правилом

$$
\lambda f\left(m_{1}, \ldots, m_{q+1}\right)=f\left(m_{2}, \ldots, m_{q+1}\right)
$$

для всех $f \in \Phi^{q}, m_{1}, \ldots, m_{q+1} \in \mathbb{L}, q \in \mathbb{Z}_{+}$.

Линейные отображения $\Delta_{\alpha}: \Phi \rightarrow \Phi, \alpha \in[1, D]$, зададим правилом

$$
\Delta_{\alpha} f\left(m_{1}, \ldots, m_{q}\right)=f\left(m_{1}+e_{\alpha}, m_{2}, \ldots, m_{q}\right)-f\left(m_{1}, m_{2}, \ldots, m_{q}\right)
$$

для всех $f \in \Phi^{q}, m_{1}, \ldots, m_{q} \in \mathbb{L}, q \in \mathbb{Z}_{+}$. В частности, $\Delta_{\alpha}\left(\Phi^{0}\right)=0$.

Линейные отображения $\lambda^{\alpha}=\xi^{\alpha} \otimes: \Phi \rightarrow \Phi, \alpha \in[1, D]$, зададим правилом

$$
\lambda^{\alpha} f\left(m_{1}, \ldots, m_{q+1}\right)=\mu_{1}^{\alpha} f\left(m_{2}, \ldots, m_{q+1}\right)
$$

для всех $f \in \Phi^{q}, m_{1}, \ldots, m_{q+1} \in \mathbb{L}, q \in \mathbb{Z}_{+}$.

Положим

$\rho=\lambda \circ \varkappa, R=\mathrm{id}-\rho$ (заметим, что $\varkappa \circ \lambda=\mathrm{id}$ );

$\varkappa_{\alpha}=\varkappa \circ \Delta_{\alpha}, \alpha \in[1, D]$

$\sigma=\sum_{1 \leqslant \alpha \leqslant D} \lambda^{\alpha} \circ \varkappa_{\alpha}, S=R-\sigma=\mathrm{id}-\rho-\sigma ;$

$\tau=\sum_{1 \leqslant \beta \leqslant D} \lambda^{\beta} \circ \Delta_{\beta}$.

ПРЕДЛОЖениЕ 4.2. Справедливы следующие равенства:

1) $d \circ \varkappa+\varkappa \circ d=\rho$;

2) $d \circ \lambda+\lambda \circ d=\lambda \circ \lambda$;

3) $d \circ \rho-\rho \circ d=0$.

ПРЕДЛОЖЕНИЕ 4.3. Для любых $\alpha, \beta \in[1, D]$ справедливы равенства

1) $\Delta_{\alpha} \circ \lambda^{\beta}=\delta_{\alpha}^{\beta} \lambda$, где $\delta_{\alpha}^{\beta}-$ символ Кронекера;

2) $\varkappa_{\alpha} \circ \Delta_{\beta}=\varkappa_{\circ} \Delta_{\alpha} \circ \Delta_{\beta}=\varkappa_{\beta} \circ \Delta_{\alpha}=\varkappa_{\alpha \beta}$;

3) $\varkappa_{\alpha} \circ \lambda^{\beta}=\delta_{\alpha}^{\beta}$ id.

ПРЕДЛОЖЕНИЕ 4.4. Для любого $\alpha \in[1, D]$ справедливы равенства

1) $d \circ \Delta_{\alpha}-\Delta_{\alpha} \circ d=\lambda \circ \Delta_{\alpha}=\Delta_{\alpha} \circ \tau$;

2) $d \circ \lambda^{\alpha}+\lambda^{\alpha} \circ d=0$

3) $d \circ \varkappa_{\alpha}+\varkappa_{\alpha} \circ d=-R \circ \Delta_{\alpha}=-\Delta_{\alpha} \circ S$;

4) $d \circ \sigma-\sigma \circ d=\tau \circ S$;

5) $\lambda \circ \varkappa_{\alpha}=\rho \circ \Delta_{\alpha}=\Delta_{\alpha} \circ \sigma$.

Доказательства этих предложений проводятся последовательными прямыми вычислениями. 
4.3. Симметрическая группа. Напомним элементарные свойства симметрической группы $\mathfrak{S}_{q}, q \in \mathbb{N}$, т.е. группы подстановок множества $[1, q]$.

1. Определен морфизм групп sign: $\mathfrak{S}_{q} \rightarrow\{ \pm 1\}, \pi \mapsto \operatorname{sign} \pi-$ знак подстановки $\pi$.

2. Подстановка $\varepsilon$ называется элементарной, если она меняет местами два соседних индекса, а остальные оставляет на своих местах. Для всякой подстановки $\pi \in \mathfrak{S}_{q}$ существуют элементарные подстановки $\varepsilon_{1}, \ldots, \varepsilon_{n} \in \mathfrak{S}_{q}, n=n(\pi)$, такие что $\pi=\varepsilon_{1} \circ \cdots \circ \varepsilon_{n}$, $\operatorname{sign} \pi=(-1)^{n}$.

3. Определено действие группы $\mathfrak{S}_{q}$ на множестве $[1, D]_{q}, \mathfrak{S}_{q} \ni \pi:[1, D]_{q} \rightarrow[1, D]_{q}$, $\mathrm{A}=\left(\alpha_{1}, \ldots, \alpha_{q}\right) \mapsto \pi \mathrm{A}=\left(\alpha_{\pi(1)}, \ldots, \alpha_{\pi(q)}\right)$.

4. Пусть $\mathcal{K}$ - линейное пространство и $\mathcal{K}^{[1, D]_{q}}=\left\{\phi=\left(\phi_{\mathrm{A}}\right):[1, D]_{q} \rightarrow \mathcal{K}\right\}-$ множество отображений из $[1, D]_{q}$ в $\mathcal{K}$. Определено действие группы $\mathfrak{S}_{q}$ на $\mathcal{K}[1, D]_{q}, \mathfrak{S}_{q} \ni$ $\pi: \mathcal{K}^{[1, D]_{q}} \rightarrow \mathcal{K}^{[1, D]_{q}}, \phi \mapsto \pi \phi,(\pi \phi)_{\mathrm{A}}=\phi_{\pi \mathrm{A}}$

5. Определена кососимметризация $\mathfrak{a}$ :

$$
\mathcal{K}^{[1, D]_{q}} \rightarrow \mathcal{K}^{[1, D]_{q}}, \quad \phi \mapsto \mathfrak{a} \phi=\frac{1}{q !} \sum_{\pi \in \mathfrak{S}_{q}} \operatorname{sign} \pi \cdot \pi \phi .
$$

Легко проверяются следующие свойства кососимметризации:

$\mathfrak{a} \circ \mathfrak{a}=\mathfrak{a}$, т.е. $\mathfrak{a}-$ проектор;

$\mathfrak{a}(\pi \phi)=\operatorname{sign} \pi \cdot \mathfrak{a} \phi$ для всех $\phi \in \mathcal{K}^{[1, D]_{q}}$ и $\pi \in \mathfrak{S}_{q}$.

6. Для любых $\phi \in \mathcal{K}^{[1, D]_{q}}$ и $\pi \in \mathfrak{S}_{q}$ сушествуют $\phi_{1}, \ldots, \phi_{n} \in \mathcal{K}^{[1, D]_{q}}$ и $\varepsilon_{1}, \ldots, \varepsilon_{n} \in$ $\mathfrak{S}_{q}, \varepsilon_{r} \phi_{r}=\phi_{r}, 1 \leqslant r \leqslant n$, такие что

$$
\phi-\operatorname{sign} \pi \cdot \pi \phi=\sum_{1 \leqslant r \leqslant n} \phi_{r} .
$$

7. Для любого $\phi \in \mathcal{K}^{[1, D]_{q}}$ сушествуют $\phi_{1}, \ldots, \phi_{N} \in \mathcal{K}^{[1, D]_{q}}$ и $\varepsilon_{1}, \ldots, \varepsilon_{N} \in \mathfrak{S}_{q}$, $\varepsilon_{r} \phi_{r}=\phi_{r}, 1 \leqslant r \leqslant N$, такие что

$$
\phi-\mathfrak{a} \phi=\sum_{1 \leqslant r \leqslant N} \phi_{r}
$$

4.4. Коцепи. Перед чтением этого пункта полезно ознакомиться с разделом 6. Линейное пространство коцепей на решетке $\mathbb{L}$ с коэффициентами из $\Phi$ есть

$$
\mathcal{C}^{* *}(\mathbb{L} ; \Phi)=\Phi \otimes_{\mathbb{Z}}\left(\wedge \mathbb{L}^{\prime}\right)=\bigoplus_{0 \leqslant p, q<\infty} \mathcal{C}^{p q}(\mathbb{L} ; \Phi)=\bigoplus_{0 \leqslant p, q<\infty} \Phi^{p} \otimes_{\mathbb{Z}}\left(\wedge^{q} \mathbb{L}^{\prime}\right) .
$$

Каждая коцеп $f \in \mathcal{C}^{p q}(\mathbb{L} ; \Phi)$ имеет однозначное представление

$$
f=\frac{1}{q !} \sum_{|\mathrm{A}|=q} f_{\mathrm{A}} \otimes e^{\mathrm{A}},
$$

или коротко, $f=\left(f_{\mathrm{A}}\right)$, где компоненты $f_{\mathrm{A}} \in \Phi^{p}$. В частности, $\mathcal{C}^{p 0}(\mathbb{L} ; \Phi)=\Phi^{p}$, $p \in \mathbb{Z}_{+}$. Введенные в п. 4.2 отображения $X=d, \varkappa, \lambda, \Delta_{\alpha}, \lambda^{\alpha}: \Phi \rightarrow \Phi$ переносятся на $\mathcal{C}^{* *}(\mathbb{L} ; \Phi)$ отождествлением $X \equiv X \otimes_{\mathbb{Z}} \mathrm{id}_{\wedge}\left(\mathbb{L}^{\prime}\right)$. Кограничный оператор $d_{\mathrm{R}}: \mathcal{C}^{p q}(\mathbb{L} ; \Phi) \rightarrow$ $\mathcal{C}^{p, q+1}(\mathbb{L} ; \Phi)$ действует по формуле

$$
\left(d_{\mathrm{R}} f\right)_{\mathrm{A}}=\sum_{0 \leqslant i \leqslant q}(-1)^{i} \Delta_{\alpha_{i}} f_{\mathrm{A}^{i}},
$$

для всех $f \in \mathcal{C}^{p q}(\mathbb{L} ; \Phi), \mathrm{A}=\left(\alpha_{0}, \ldots, \alpha_{q}\right)$. 
ПРЕДЛОЖЕНИЕ 4.5. Справедливы равенства

1) $d \circ d_{\mathrm{R}}=d_{\mathrm{R}} \circ(d+\tau)$

2) $\varkappa \circ(d+\tau)+(d-\lambda) \circ \varkappa=0$;

3) $\varkappa_{\alpha} \circ(d+\tau)+(d-\lambda) \circ \varkappa_{\alpha}=0$ для всех $\alpha \in[1, D]$.

Доказывается прямыми вычислениями с использованием предложений 4.2-4.4.

Линейное отображение $\mathfrak{h}: \mathcal{C}^{*} \rightarrow \mathcal{C}^{*}$, введенное в п. 6.6 , очевидным образом переносится на $\mathcal{C}^{* *}(\mathbb{L} ; \Phi)$. Из явного вида этого отображения следует, что если компоненты $f_{\mathrm{A}} \in \Phi^{p}$, то и компоненты $(\mathfrak{h} f)_{\mathrm{A}} \in \Phi^{p}$. Далее, легко проверяется, что если компоненты $f_{\mathrm{A}}(m, \cdot)=0$ для всех $m \in \mathbb{L}_{+},|m| \leqslant r$, то компоненты $(\mathfrak{h} f)_{\mathrm{A}}(m, \cdot)=0$ для всех $m \in \mathbb{L}_{+}$, $|m| \leqslant r+1$, а компоненты $\left(d_{\mathrm{R}} f\right)_{\mathrm{A}}(m, \cdot)=0$ для всех $m \in \mathbb{L}_{+},|m| \leqslant r-1$, где значок заменяет остальные аргументы $m_{2}, \ldots, m_{p} \in \mathbb{L}$, которые могут принимать произвольные значения. Заметим, что условие $f_{\mathrm{A}}(m, \cdot)=0$ для $|m|=0$ эквивалентно условию $\varkappa f_{\mathrm{A}}=0$, а условие $f_{\mathrm{A}}(m, \cdot)=0$ для всех $m \in \mathbb{L}_{+}, \quad|m| \leqslant 1$, эквивалентно условию $\varkappa f_{\mathrm{A}}=\varkappa_{\alpha} f_{\mathrm{A}}=0$ для всех $\alpha \in[1, D]$, которое, в свою очередь, эквивалентно условию $S f_{\mathrm{A}}=f_{\mathrm{A}}$.

Лемма 4.1. Пусть $f \in \mathcal{C}^{p q}(\mathbb{L} ; \Phi), d_{\mathrm{R}} f=0, S f=f, p \in \mathbb{N}, q \in \mathbb{Z}_{+}$. Тогда $f=0$ при $q=0$ u $f=d_{\mathrm{R}} g$ при $q \in \mathbb{N}$, где $g \in \mathcal{C}^{p, q-1}(\mathbb{L} ; \Phi), \quad g(m, \cdot)=0$ для всех $m \in \mathbb{L}_{+}$, $|m| \leqslant 2$.

ДокАЗАТЕЛЬСТво. Следует воспользоваться доказательством леммы 6.1 и учесть приведенные выше рассуждения.

\section{5. ВЫЧИСЛЕНИЕ КОГОМОЛОГИЙ}

5.1. Подготовительные леммы. Введем ряд обозначений. Для $f, g \in \Phi^{q}, q \in$ $\mathbb{N}$, будем писать $f \sim g$, если $f-g=d h$, для некоторого $h \in \Phi^{q-1}$. Далее, для $\mathrm{A}=$ $\left(\alpha_{1}, \ldots, \alpha_{q}\right), q \in \mathbb{N}$, положим $(\otimes \xi)^{\mathrm{A}}=\xi^{\alpha_{1}} \otimes \cdots \otimes \xi^{\alpha_{q}} \in \Phi^{q}$ и $(\circ \varkappa)_{\mathrm{A}}=\varkappa_{\alpha_{1}} \circ \cdots \circ \varkappa_{\alpha_{q}}$.

Лемма 5.1. Справедливо равенство $d(\otimes \xi)^{\mathrm{A}}=0$, в частности, $f \sim g$ влечет $(\otimes \xi)^{\mathrm{A}} \otimes f \sim(\otimes \xi)^{\mathrm{A}} \otimes g$, для всех $\mathrm{A} \in[1, D]_{p}, \quad f, g \in \Phi^{q}, \quad p, q \in \mathbb{N}$.

ДокАЗАТЕльСтво. Следует воспользоваться предложением 4.1 и проверить, что $d \xi^{\alpha}=0, \alpha \in[1, D]$.

ЛЕмМа 5.2. Пусть

$$
f=\sum_{|\mathrm{A}|=p}(\otimes \xi)^{\mathrm{A}} \otimes g_{\mathrm{A}^{\prime}} \in \Phi^{q}, \quad g_{\mathrm{A}} \in \Phi^{q-p}, \quad p \leqslant q
$$

mогда $g_{\mathrm{A}}=(\circ \varkappa)_{\mathrm{A}} f$.

ДокАЗАТЕЛЬСтво. Достаточно воспользоваться равенством $\varkappa_{\alpha} \xi^{\beta}=\delta_{\alpha}^{\beta}$ для всех $\alpha, \beta \in[1, D]$ (см. предложение 4.3(3)). 
Лемма 5.3. Пусть

$$
f=\sum_{1 \leqslant \alpha, \beta \leqslant D} \xi^{\alpha} \otimes \xi^{\beta} \otimes g_{\alpha \beta} \in \Phi^{q},
$$

где $g_{\alpha \beta} \in \Phi^{q-2}$, причем $g_{\alpha \beta}=g_{\beta \alpha}$. Тогда $f \sim h$, где $h \in \Phi^{q}, \quad S h=h$.

ДокАЗАтЕльство. Зададим функции $\xi^{\alpha \beta} \in \Phi^{1}, \quad \alpha, \beta \in[1, D]$, равенствами $\xi^{\alpha \beta}(m)=\mu^{\alpha} \mu^{\beta}-\delta_{\alpha}^{\beta} \mu^{\alpha}$ для всех $m=\left(\mu^{1}, \ldots, \mu^{D}\right) \in \mathbb{L}$. С помошью предложения 4.1 легко проверяется, что в условиях леммы

$$
f=d\left(-\frac{1}{2} \sum \xi^{\alpha \beta} \otimes g_{\alpha \beta}\right)+h
$$

где $h=\frac{1}{2} \sum \xi^{\alpha \beta} \otimes d g_{\alpha \beta}$, сумма берется по всем $\alpha, \beta \in[1, D]$. Для завершения доказательства заметим, что $\varkappa^{\alpha \beta}=\varkappa_{\gamma} \xi^{\alpha \beta}=0$ для всех $\alpha, \beta, \gamma \in[1, D]$.

Лемма 5.4. Пусть $f \in \Phi^{q}, d f=0, q \in \mathbb{N}$, тогда

$$
f \sim \sum_{|\mathrm{A}|=q}(\otimes \xi)^{\mathrm{A}} \cdot c_{\mathrm{A}^{\prime}}+\sum_{|\mathrm{B}| \leqslant q-1}(\otimes \xi)^{\mathrm{B}} \otimes g_{\mathrm{B}^{\prime}}
$$

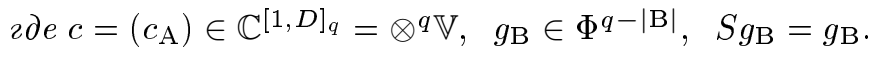

ДокАЗАТЕльСтво. Используем метод индукции. Пусть $f \in \Phi^{q}, d f=0$. Тогда в силу предложения $4.2(1)$

$$
f=\rho f+R f=d(\varkappa f)+\varkappa(d f)+R f \sim R f=\sigma f+S f=\sum_{1 \leqslant \alpha \leqslant D} \xi^{\alpha} \otimes f_{\alpha}+g,
$$

где $f_{\alpha}=\varkappa_{\alpha} f \in \Phi^{q-1}, g=S f \in \Phi^{q}$, причем $S g=g$, поскольку $S \circ S=S$. Предположим теперь, что для $r<q$ справедливо представление

$$
f \sim \tilde{f}=\sum_{|\mathrm{A}|=r}(\otimes \xi)^{\mathrm{A}} \otimes f_{\mathrm{A}^{\prime}}+\sum_{|\mathrm{B}| \leqslant r-1}(\otimes \xi)^{\mathrm{B}} \otimes g_{\mathrm{B}^{\prime}},
$$

где $f_{\mathrm{A}} \in \Phi^{q-r}, g_{\mathrm{B}} \in \Phi^{q-|\mathrm{B}|}, S g_{\mathrm{B}}=g_{\mathrm{B}}$, и докажем, что такое же представление справедливо и для $r+1$. С учетом предложения 4.1 и леммы 5.1 имеем

$$
0=d \tilde{f}=(-1)^{r} \sum_{|\mathrm{A}|=r}(\otimes \xi)^{\mathrm{A}} \otimes d f_{\mathrm{A}^{\prime}}+\sum_{|\mathrm{B}| \leqslant r-1}(-1)^{|\mathrm{B}|}(\otimes \xi)^{\mathrm{B}} \otimes d g_{\mathrm{B}^{\prime}} .
$$

Пусть $\mathrm{A}=\left(\alpha_{1}, \ldots, \alpha_{r}\right)$. Действуя на предыдущее равенство оператором $(\text { о })_{\mathrm{A}^{\prime}}$, с помошью леммы 5.2 получаем

$$
(-1)^{r} d f_{\mathrm{A}^{\prime}}+\sum_{0 \leqslant p \leqslant r-1}(-1)^{p} \varkappa_{\alpha_{r}} \ldots \varkappa_{\alpha_{p+1}} d g_{\alpha_{p} \ldots \alpha_{1}}=0
$$


или, в силу предложения $4.4(3)$ и равенства $S g_{\mathrm{B}}=g_{\mathrm{B}}$,

$$
(-1)^{r}\left(d f_{\mathrm{A}^{\prime}}+\Delta_{\alpha_{r}} g_{\alpha_{r-1} \ldots \alpha_{1}}\right)+\sum_{0 \leqslant p \leqslant r-2}(-1)^{p+1} \varkappa_{\alpha_{r}} \ldots \varkappa_{\alpha_{p+3}} \varkappa_{\alpha_{p+2} \alpha_{p+1}} g_{\alpha_{p} \ldots \alpha_{1}}=0
$$

где $\varkappa_{\alpha_{p+2} \alpha_{p+1}}=\varkappa \circ \Delta_{\alpha_{p+2}} \circ \Delta_{\alpha_{p+1}}=\varkappa_{\alpha_{p+1} \alpha_{p+2}}$ (см. предложение 4.3(2)). Действуя на это равенство оператором $\varkappa$ и учитывая предложение $4.2(1)$, находим

$$
\rho f_{\mathrm{A}^{\prime}} \sim \sum_{0 \leqslant p \leqslant r-2}(-1)^{r-p} \varkappa_{\alpha_{r}} \ldots \varkappa_{\alpha_{p+3}} \varkappa_{\alpha_{p+2} \alpha_{p+1}} g_{\alpha_{p} \ldots \alpha_{1}}
$$

где учтено, что $\varkappa_{\alpha} g_{\mathrm{B}}=0$ для всех $\alpha \in[1, D]$. Таким образом,

$$
f_{\mathrm{A}^{\prime}}=\rho f_{\mathrm{A}^{\prime}}+\sum_{1 \leqslant \alpha \leqslant D} \xi^{\alpha} \otimes \tilde{f}_{\alpha \mathrm{A}^{\prime}}+S f_{\mathrm{A}^{\prime}} \sim \sum_{1 \leqslant \alpha \leqslant D} \xi^{\alpha} \otimes \tilde{f}_{\alpha \mathrm{A}^{\prime}}+\tilde{g}_{\mathrm{A}^{\prime}}+\sum_{0 \leqslant p \leqslant r-2} h_{\mathrm{A}^{\prime}}^{(p)},
$$

где $\tilde{f}_{\alpha \mathrm{A}^{\prime}}=\varkappa_{\alpha} f_{\mathrm{A}^{\prime}}, \quad \tilde{g}_{\mathrm{A}^{\prime}}=S f_{\mathrm{A}^{\prime}}, h_{\mathrm{A}^{\prime}}^{(p)}=(-1)^{r-p} \varkappa_{\alpha_{r}} \ldots \varkappa_{\alpha_{p+3}} \varkappa_{\alpha_{p+2} \alpha_{p+1}} g_{\alpha_{p} \ldots \alpha_{1}}$, причем $S \tilde{g}_{\mathrm{A}^{\prime}}=\tilde{g}_{\mathrm{A}^{\prime}}, \varepsilon_{p} h_{\mathrm{A}^{\prime}}^{(p)}=h_{\mathrm{A}^{\prime}}^{(p)}$, где элементарная подстановка $\varepsilon_{p}$ меняет местами индексы $p+1$ и $p+2$. Подставив последнее выражение в исходное представление для $\tilde{f}, \mathrm{c}$ помощью леммы 5.1 получим

$$
\begin{aligned}
f & \sim \tilde{f} \sim \sum_{|\mathrm{A}|=r} \sum_{1 \leqslant \alpha \leqslant D}(\otimes \xi)^{\mathrm{A}} \otimes \xi^{\alpha} \otimes f_{\alpha \mathrm{A}^{\prime}}+\sum_{|\mathrm{A}|=r}(\otimes \xi)^{\mathrm{A}} \otimes \tilde{g}_{\mathrm{A}^{\prime}}+ \\
& +\sum_{0 \leqslant p \leqslant r-2} \sum_{|\mathrm{A}|=r}(\otimes \xi)^{\mathrm{A}} \otimes h_{\mathrm{A}^{\prime}}^{(p)}+\sum_{|\mathrm{B}| \leqslant r-1}(\otimes \xi)^{\mathrm{B}} \otimes g_{\mathrm{B}^{\prime}} \sim \\
& \sim \sum_{|\mathrm{A}|=r+1}(\otimes \xi)^{\mathrm{A}} \otimes \tilde{f}_{\mathrm{A}^{\prime}}+\sum_{|\mathrm{B}| \leqslant r}(\otimes \xi)^{\mathrm{B}} \otimes \tilde{g}_{\mathrm{B}^{\prime}},
\end{aligned}
$$

где $\tilde{g}_{\mathrm{B}}=g_{\mathrm{B}}$ при $|\mathrm{B}|=r-1, \tilde{g}_{\mathrm{B}}=g_{\mathrm{B}}+\tilde{h}_{\mathrm{B}}^{(|\mathrm{B}|)}$ при $|\mathrm{B}| \leqslant r-2$, функции $\tilde{h}_{\mathrm{B}}^{(|\mathrm{B}|)}$ вычисляются с помощью леммы 5.3 с учетом равенства $\varepsilon_{p} h_{\mathrm{A}^{\prime}}^{(p)}=h_{\mathrm{A}^{\prime}}^{(p)}$. По построению $S \tilde{g}_{\mathrm{B}}=\tilde{g}_{\mathrm{B}}$, так что требуемое представление для $r+1$ получено. Полагая теперь $r=q$ и учитывая, что $\Phi^{0}=\mathbb{C}$, завершаем доказательство леммы.

ЛеммА 5.5. Пусть $c=\left(c_{\mathrm{A}}\right) \in \otimes q \mathbb{V}$, тогда

$$
\sum_{|\mathrm{A}|=q}(\otimes \xi)^{\mathrm{A}} \cdot c_{\mathrm{A}^{\prime}} \sim \sum_{|\mathrm{A}|=q}(\wedge \xi)^{\mathrm{A}} \cdot v_{\mathrm{A}^{\prime}}
$$

где значок $\wedge$ означает внешнее (кососимметризованное) произведение, $v=\left(v_{\mathrm{A}}\right)=$ $\mathfrak{a} c \in \wedge^{q} \mathbb{V}$.

ДокАЗАТЕЛЬСтво. Следует воспользоваться свойством 7 симметрической группы и подходящей модификацией леммы 5.3. 
Лемма 5.6. Пусть $g_{\mathrm{B}} \in \Phi^{q-|\mathrm{B}|}, S g_{\mathrm{B}}=g_{\mathrm{B}},|\mathrm{B}| \leqslant q-1$, тогдa

$$
f=\sum_{|\mathrm{B}| \leqslant q-1}(\otimes \xi)^{\mathrm{B}} \otimes g_{\mathrm{B}^{\prime}} \sim \tilde{f}=\sum_{|\mathrm{B}| \leqslant q-1}(\wedge \xi)^{\mathrm{B}} \otimes \tilde{g}_{\mathrm{B}^{\prime}},
$$

əде $\tilde{g}_{\mathrm{B}} \in \Phi^{q-|\mathrm{B}|}, \quad S \tilde{g}_{\mathrm{B}}=\tilde{g}_{\mathrm{B}}, \quad(\mathfrak{a} \tilde{g})_{\mathrm{B}}=\tilde{g}_{\mathrm{B}}, \quad|\mathrm{B}| \leqslant q-1$.

ДоКАЗАТЕЛЬСТВо. Пусть сначала $|\mathrm{B}|=q-1$. В силу свойства 7 симметрической группы

$$
\sum_{|\mathrm{B}|=q-1}(\otimes \xi)^{\mathrm{B}} \otimes g_{\mathrm{B}^{\prime}}=\sum_{|\mathrm{B}|=q-1}(\otimes \xi)^{\mathrm{B}} \otimes\left((\mathfrak{a} g)_{\mathrm{B}^{\prime}}+\sum_{1 \leqslant r \leqslant N} g_{r \mathrm{~B}^{\prime}}\right),
$$

где $g_{r \mathrm{~B}} \in \Phi^{q-|\mathrm{B}|}, S g_{r \mathrm{~B}}=g_{r \mathrm{~B}},\left(\varepsilon_{r} g_{r}\right)_{\mathrm{B}}=g_{r \mathrm{~B}}$ для некоторых элементарных подстановок $\varepsilon_{r}, 1 \leqslant r \leqslant N$. С помошью леммы 5.3 получаем

$$
\sum_{|\mathrm{B}|=q-1}(\otimes \xi)^{\mathrm{B}} \otimes g_{r \mathrm{~B}^{\prime}} \sim \sum_{|\mathrm{B}|=s-1}(\otimes \xi)^{\mathrm{B}} \otimes h_{r \mathrm{~B}^{\prime}}
$$

где $s=s(r)<q-1, h_{r \mathrm{~B}} \in \Phi^{q-|\mathrm{B}|}, S h_{r \mathrm{~B}}=h_{r \mathrm{~B}}$, откуда

$$
f \sim \sum_{|\mathrm{B}|=q-1}(\wedge \xi)^{\mathrm{B}} \otimes \tilde{g}_{\mathrm{B}^{\prime}}+\sum_{|\mathrm{B}| \leqslant q-2}(\otimes \xi)^{\mathrm{B}} \otimes g_{\mathrm{B}^{\prime}}^{\prime}
$$

где $\tilde{g}=\mathfrak{a} g, g_{\mathrm{B}}^{\prime} \in \Phi^{q-|\mathrm{B}|}, S g_{\mathrm{B}}^{\prime}=g_{\mathrm{B}}^{\prime}$. Повторяя эти рассуждения для $|\mathrm{B}|=q-2, q-3, \ldots$, завершаем доказательство.

ЛЕмма 5.7. Пусть

$$
f=\sum_{|\mathrm{B}| \leqslant q-1}(\wedge \xi)^{\mathrm{B}} \otimes g_{\mathrm{B}^{\prime}}
$$

где $g_{\mathrm{B}} \in \Phi^{q-|\mathrm{B}|}, \quad S g_{\mathrm{B}}=g_{\mathrm{B}}$, причем $d f=0$. Тогда $f \sim 0$.

ДокАЗАТЕльСТво. Для удобства положим $g_{\mathrm{B}}^{(p)}=\left\{g_{\mathrm{B}} ;|\mathrm{B}|=p\right\}$, так что $g^{(p)}=$ $\left(g_{\mathrm{B}}^{(p)}\right) \in \mathcal{C}^{q-p, p}(\mathbb{L} ; \Phi), S g^{(p)}=g^{(p)}$. Условие $d f=0$ запишется в виде

$$
\sum_{0 \leqslant p \leqslant q-1}(-1)^{p} \sum_{|\mathrm{B}|=p}(\wedge \xi)^{\mathrm{B}} \otimes d g_{\mathrm{B}^{\prime}}^{(p)}=0 .
$$

Действуя на это равенство оператором $(\circ \varkappa)_{\mathrm{A}^{\prime}}, \mathrm{A}=\left(\alpha_{1}, \ldots, \alpha_{q}\right)$, получаем

$$
(-1)^{q} \Delta_{\alpha_{q}} g_{\alpha_{q-1} \ldots \alpha_{1}}^{(q-1)}+\sum_{0 \leqslant p \leqslant q-2}(-1)^{p+1} \varkappa_{\alpha_{q}} \ldots \varkappa_{\alpha_{p+3}} \varkappa_{\alpha_{p+2} \alpha_{p+1}} g_{\alpha_{p} \ldots \alpha_{1}}^{(p)}=0,
$$

где мы учли предложения 4.4(3) и 4.3(2). Кососимметризуя это равенство, получим $d_{\mathrm{R}} g^{(q-1)}=0$, поскольку второе слагаемое содержит операторы $\varkappa_{\alpha_{p+2} \alpha_{p+1}}=\varkappa_{\alpha_{p+1} \alpha_{p+2}}$. В силу леммы $4.1 g^{(q-1)}=d_{\mathrm{R}} h^{(1)}$, где $h^{(1)} \in \mathcal{C}^{1, q-2}(\mathbb{L} ; \Phi)$, причем $h^{(1)}(m)=0$ при 
$m \in \mathbb{L}_{+},|m| \leqslant 2$. Подставляя это представление в выражение для $d f=0$ и действуя оператором $(\circ \varkappa)_{\mathrm{A}^{\prime}}, \mathrm{A}=\left(\alpha_{1}, \ldots, \alpha_{q-1}\right)$, получим

$$
(-1)^{q-1} d\left(d_{\mathrm{R}} h^{(1)}\right)_{\mathrm{A}^{\prime}}+(-1)^{q-2+1} \Delta_{\alpha_{q-1}} g_{\alpha_{q-2} \ldots \alpha_{1}}^{(q-2)}+\cdots=0,
$$

где мы использовали предложение 4.4(3), опущенные члены содержат операторы вида $\varkappa_{\alpha \beta}$. Кососимметризуя это равенство, получим

$$
d\left(d_{\mathrm{R}} h^{(1)}\right)+d_{\mathrm{R}} g^{(q-2)}=0,
$$

и предложение $4.5(1)$ дает

$$
d_{\mathrm{R}}\left((d+\tau) h^{(1)}+g^{(q-2)}\right)=0,
$$

откуда опять в силу леммы 4.1 находим

$$
g^{(q-2)}=-(d+\tau) h^{(1)}+d_{\mathrm{R}} h^{(2)},
$$

где в силу предложения $4.5(2,3)$ коцепь $h^{(2)} \in \mathcal{C}^{2, q-3}(\mathbb{L} ; \Phi), h^{(2)}(m, \cdot)=0$ при $m \in \mathbb{L}_{+}$, $|m| \leqslant 2$. Исходное выражение для $f$ теперь принимает вид

$$
\begin{aligned}
f= & \sum_{|\mathrm{B}|=q-1}(\wedge \xi)^{\mathrm{B}} \otimes\left(d_{\mathrm{R}} h^{(1)}\right)_{\mathrm{B}^{\prime}}+ \\
& +\sum_{|\mathrm{B}|=q-2}(\wedge \xi)^{\mathrm{B}} \otimes\left(-(d+\tau) h^{(1)}+d_{\mathrm{R}} h^{(2)}\right)_{\mathrm{B}^{\prime}}+\sum_{|\mathrm{B}| \leqslant q-3}(\wedge \xi)^{\mathrm{B}} \otimes g_{\mathrm{B}^{\prime}}^{(|\mathrm{B}|)} \sim \\
\sim & \left(\sum_{|\mathrm{B}|=q-1}(\wedge \xi)^{\mathrm{B}} \otimes\left(d_{\mathrm{R}} h^{(1)}\right)_{\mathrm{B}^{\prime}}-\sum_{|\mathrm{B}|=q-2} \sum_{1 \leqslant \beta \leqslant D}(\wedge \xi)^{\mathrm{B}} \otimes \xi^{\beta} \otimes \Delta_{\beta} h_{\mathrm{B}^{\prime}}^{(1)}\right)+ \\
& +\sum_{|\mathrm{B}|=q-2}(\wedge \xi)^{\mathrm{B}} \otimes\left(d_{\mathrm{R}} h^{(2)}\right)_{\mathrm{B}^{\prime}}+\sum_{|\mathrm{B}| \leqslant q-3}(\wedge \xi)^{\mathrm{B}} \otimes g_{\mathrm{B}^{\prime}}^{(|\mathrm{B}|)} \sim \\
\sim & \sum_{|\mathrm{B}|=q-2}(\wedge \xi)^{\mathrm{B}} \otimes\left(d_{\mathrm{R}} h^{(2)}\right)_{\mathrm{B}^{\prime}}+\sum_{|\mathrm{B}| \leqslant q-3}(\wedge \xi)^{\mathrm{B}} \otimes \tilde{g}_{\mathrm{B}^{\prime}}^{(|\mathrm{B}|)},
\end{aligned}
$$

где мы учли лемму 5.6 при вычислении выражения в больших круглых скобках. Повторяя эти рассуждения для $|\mathrm{B}|=q-2, q-3, \ldots$, завершаем доказательство. (Последний шаг $|\mathrm{B}|=1$ требует легкой модификкации.)

5.2. Когомологии вспомогательного комплекса. Вычислим пространства когомологий $H^{q}(\Phi)$ вспомогательного комплекса $\{\Phi ; d\}$.

Теорема 5.1. Справедливы равенства

$$
H^{q}(\Phi)=\wedge^{q} \mathbb{V}, \quad q \in \mathbb{Z}_{+},
$$

где $\wedge^{q} \mathbb{V}$ есть $q$-я внешняя степень комплексного векторного пространства $\mathbb{V}$ (в частности, $\wedge^{0} \mathbb{V}=\mathbb{C}, \wedge^{q} \mathbb{V}=0$ при $q>D$ ). 
ДокАЗАТЕльство. Достаточно последовательно воспользоваться леммами 5.4-5.7 и убедиться, что для любого $v \in \wedge^{q} \mathbb{V}$ функция

$$
f=\sum_{|\mathrm{A}|=q}(\wedge \xi)^{\mathrm{A}} v_{\mathrm{A}^{\prime}}
$$

удовлетворяет равенству $d f=0$, причем $f=d g, g \in \Phi^{q-1}$, лишь в случае $v=0, g=0$.

5.3. Когомологии алгебры гладких функций на торе. Основной результат работы составляет

Теорема 5.2. Имеет место изоморфизм

$$
H(\mathcal{E}(\mathbb{T}) ; \mathcal{E}(\mathbb{T})) \simeq \mathcal{E}(\mathbb{T}) \otimes(\wedge \mathbb{V})
$$

где $\ \mathbb{V}$ - внешняя алгебра векторного пространства $\mathbb{V}$.

ДокАЗАТЕЛЬСТво. Для доказательства следует воспользоваться предложением 3.1 и теоремой 5.1 .

\section{6. ДОПОЛНЕНИЕ}

В этом разделе мы вычисляем когомологии комплекса де Рама на целочисленной решетке, причем, учитывая популярность решетчатых моделей, даем краткое введение в предмет (см. также, например, [6], [7]).

6.1. Функции на решетке. Напомним, что иелочисленная решетка (далее решетка) $\mathbb{L}=\mathbb{Z}^{D}, D \in \mathbb{N}$, так что для каждого $m=\left(\mu^{1}, \ldots, \mu^{D}\right) \in \mathbb{L}$ имеем

$$
m=\sum_{1 \leqslant \alpha \leqslant D} \mu^{\alpha} e_{\alpha}
$$

Обозначим через $\mathcal{F}=\{f: \mathbb{L} \rightarrow \mathbb{C}\}$ линейное пространство всех комплексных функций на $\mathbb{L}$, и пусть $\mathcal{F}_{0}$ - подпространство всех функций с конечными носителями, где носитель функции $f \in \mathcal{F}$ есть множество $\operatorname{supp} f=\{m \in \mathbb{L}: f(m) \neq 0\}$. Для каждой точки $m \in \mathbb{L}$ определена функция $\delta_{m} \in \mathcal{F}$ правилом $\delta_{m}(n)=1$ при $n=m$ и $\delta_{m}(n)=0$ при $n \neq m$, $n \in \mathbb{L}$, так что

$$
f=\sum_{m \in \mathbb{L}} f(m) \delta_{m}
$$

для всякой функции $f \in \mathcal{F}$. Для каждой пары $f \in \mathcal{F}, g \in \mathcal{F}_{0}$ определено естественное спаривание

$$
\langle f, g\rangle=\sum_{m \in \mathbb{L}} f(m) g(m) \in \mathbb{C},
$$

так что пространства $\mathcal{F}$ и $\mathcal{F}_{0}$ можно считать дуальными друг другу. Линейные отображения $\Delta_{\alpha}: \mathcal{F} \rightarrow \mathcal{F}, \alpha \in[1, D]$, действуют по правилу $\Delta_{\alpha} f(m)=f\left(m+e_{\alpha}\right)-f(m)$ для всех $f \in \mathcal{F}, m \in \mathbb{L}$, причем $\Delta_{\alpha}\left(\mathcal{F}_{0}\right) \subset \mathcal{F}_{0}$. В частности, $\Delta_{\alpha} \delta_{m}=\delta_{m-e_{\alpha}}-\delta_{m}$ для всех $\alpha \in[1, D], m \in \mathbb{L}$. Дуальные отображения $\nabla_{\alpha}: \mathcal{F} \rightarrow \mathcal{F}, \alpha \in[1, D]$, определяются условием $\left\langle\nabla_{\alpha} f, g\right\rangle=\left\langle f, \Delta_{\alpha} g\right\rangle$ для всех $f \in \mathcal{F}, g \in \mathcal{F}_{0}$ и действуют по правилу $\nabla_{\alpha} f(m)=f\left(m-e_{\alpha}\right)-f(m)$ для всех $f \in \mathcal{F}, m \in \mathbb{L}$, так что $\nabla_{\alpha}\left(\mathcal{F}_{0}\right) \subset \mathcal{F}_{0}$. В частности, $\nabla_{\alpha} \delta_{m}=\delta_{m+e_{\alpha}}-\delta_{m}$ для всех $\alpha \in[1, D], m \in \mathbb{L}$. 
6.2. Внешняя алгебра решетки. На решетке $\mathbb{L}$ определена естественная структура $\mathbb{Z}$-модуля. Следовательно, определена внешняя алгебра

$$
\wedge \mathbb{L}=\sum_{0 \leqslant q<\infty} \wedge^{q} \mathbb{L},
$$

где $\wedge^{0} \mathbb{L}=\mathbb{Z} ; \wedge^{q} \mathbb{L}=\wedge_{\mathbb{Z}}^{q} \mathbb{L}, q \in \mathbb{N}$, есть $q$-я внешняя степень $\mathbb{Z}$-модуля $\mathbb{L}\left(\wedge^{q} \mathbb{L}=0\right.$ при $q>D)$. Элементы $e_{\alpha}, \alpha \in[1, D]$, образуют базис $\mathbb{Z}$-модуля $\mathbb{L} ;$ соответственно элементы $e_{\mathrm{A}}=e_{\alpha_{1}} \wedge \cdots \wedge e_{\alpha_{q}}, 1 \leqslant \alpha_{1}<\cdots<\alpha_{q} \leqslant D$, образуют базис $\mathbb{Z}$-модуля $\wedge^{q} \mathbb{L}, q \in \mathbb{N}$, так что каждый элемент $M \in \wedge^{q} \mathbb{L}, q \in \mathbb{N}$, имеет однозначное представление

$$
M=\frac{1}{q !} \sum_{|\mathrm{A}|=q} \mu^{\mathrm{A}} e_{\mathrm{A}}
$$

$\mu^{\mathrm{A}}=\mu^{\alpha_{1} \ldots \alpha_{q}} \in \mathbb{Z}$, причем $\mu^{\pi \mathrm{A}}=\operatorname{sign} \pi \mu^{\mathrm{A}}$ для всех $\pi \in \mathfrak{S}_{q}$. Аддитивные отображения $e_{\alpha}^{\wedge}: \wedge \mathbb{L} \rightarrow \wedge \mathbb{L}, \alpha \in[1, D]$, определяются условием $e_{\alpha}^{\wedge}\left(e_{\alpha_{1}} \wedge \cdots \wedge e_{\alpha_{q}}\right)=e_{\alpha} \wedge e_{\alpha_{1}} \wedge \cdots \wedge e_{\alpha_{q}}$ для всех $\alpha_{1}, \ldots, \alpha_{q} \in[1, D], q \in \mathbb{Z}_{+}$, и действуют по правилу

$$
\wedge^{q} \mathbb{L} \ni M=\frac{1}{q !} \sum_{|\mathrm{A}|=q} \mu^{\mathrm{A}} e_{\mathrm{A}} \mapsto e_{\alpha}^{\wedge} M=\frac{1}{(q+1) !} \sum_{|\mathrm{A}|=q+1}\left(e_{\alpha}^{\wedge} M\right)^{\mathrm{A}} e_{\mathrm{A}} \in \wedge^{q+1} \mathbb{L}
$$

где

$$
\left(e_{\alpha}^{\wedge} M\right)^{\mathrm{A}}=\sum_{0 \leqslant i \leqslant q}(-1)^{i} \delta_{\alpha}^{\alpha_{i}} \mu^{\mathrm{A}^{i}}, \quad \mathrm{~A}=\left(\alpha_{0}, \ldots, \alpha_{q}\right) .
$$

6.3. Дуальная внешняя алгебра. Дуальная решетка $\mathbb{L}^{\prime}=\operatorname{Hom}_{\mathbb{Z}}(\mathbb{L} ; \mathbb{Z})$ имеет дуальный базис $e^{\alpha}, \alpha \in[1, D]$, где $\left\langle e^{\alpha}, e_{\beta}\right\rangle=\delta_{\beta}^{\alpha}$ для всех $\alpha, \beta \in[1, D]$. Дуальная внешняя алгебра $(\wedge \mathbb{L})^{\prime}=\wedge \mathbb{L}^{\prime}$ имеет базис $e^{\mathrm{A}}=e^{\alpha_{1}} \wedge \cdots \wedge e^{\alpha_{q}}, 1 \leqslant \alpha_{1}<\cdots<\alpha_{q} \leqslant D, q \in \mathbb{Z}_{+}$, так что каждый элемент $N \in \wedge^{q} \mathbb{L}^{\prime}, q \in \mathbb{N}$, имеет однозначное представление

$$
N=\frac{1}{q !} \sum_{|\mathrm{A}|=q} \nu_{\mathrm{A}} e^{\mathrm{A}}
$$

$\nu_{\mathrm{A}}=\nu_{\alpha_{1} \ldots \alpha_{q}} \in \mathbb{Z}$, причем $\nu_{\pi \mathrm{A}}=\operatorname{sign} \pi \nu_{\mathrm{A}}$ для всех $\pi \in \mathfrak{S}_{q}$. Спаривание $\langle N, M\rangle \in \mathbb{Z}$ элементов

$$
M=\frac{1}{q !} \sum_{|\mathrm{A}|=q} \mu^{\mathrm{A}} e_{\mathrm{A}} \in \wedge^{q} \mathbb{L}, \quad N=\frac{1}{q !} \sum_{|\mathrm{A}|=q} \nu_{\mathrm{A}} e^{\mathrm{A}} \in \wedge^{q} \mathbb{L}^{\prime}, \quad q \in \mathbb{Z}_{+},
$$

вычисляется по формуле

$$
\langle N, M\rangle=\frac{1}{q !} \sum_{|\mathrm{A}|=q} \nu_{\mathrm{A}} \mu^{\mathrm{A}} .
$$

Аддитивные отображения $e_{\alpha}^{\wedge}: \wedge \mathbb{L} \rightarrow \wedge \mathbb{L}$ имеют дуальные отображения $\iota_{\alpha}: \wedge \mathbb{L}^{\prime} \rightarrow \wedge \mathbb{L}^{\prime}$, $\alpha \in[1, D]$, определяемые условиями $\left\langle\iota_{\alpha} N, M\right\rangle=\left\langle N, e_{\alpha}^{\wedge} M\right\rangle$ для всех $N \in \wedge^{q} \mathbb{L}^{\prime}, \quad M \in$ $\wedge^{q-1} \mathbb{L}$ и действующие по правилу

$$
\wedge^{q} \mathbb{L}^{\prime} \ni N=\frac{1}{q !} \sum_{|\mathrm{A}|=q} \nu_{\mathrm{A}} e^{\mathrm{A}} \mapsto \iota_{\alpha} N=\frac{1}{(q-1) !} \sum_{|\mathrm{A}|=q-1}\left(\iota_{\alpha} N\right)_{\mathrm{A}} e^{\mathrm{A}} \in \wedge^{q-1} \mathbb{L}^{\prime},
$$

где $\left(\iota_{\alpha} N\right)_{\mathrm{A}}=\nu_{\alpha \mathrm{A}}$. 
6.4. Цепи на решетке. Линейное пространство иепей на решетке $\mathbb{L}$ есть прямая сумма $\mathcal{C}_{*} \equiv \mathcal{C}_{*}(\mathbb{L})=\bigoplus_{0 \leqslant q<\infty} \mathcal{C}_{q}$, где линейные пространства $\mathcal{C}_{q}=\mathcal{F}_{0} \otimes(\wedge q \mathbb{L})=$ $\mathcal{F}_{0} \otimes_{\mathbb{Z}}\left(\wedge^{q} \mathbb{L}\right) \quad\left(\mathcal{C}_{0}=\mathcal{F}_{0}, \mathcal{C}_{q}=0\right.$ при $\left.q>D\right)$. Базис в $\mathcal{C}_{q}, q \in \mathbb{Z}_{+}$, образуют $q$-клетки $\delta_{m} \otimes e_{\mathrm{A}}, \quad m \in \mathbb{L}, \quad \mathrm{A}=\left(\alpha_{1}, \ldots, \alpha_{q}\right), \quad 1 \leqslant \alpha_{1}<\cdots<\alpha_{q} \leqslant D$, так что каждая $q$-цепь $c \in \mathcal{C}_{q}$ имеет однозначное представление

$$
c=\frac{1}{q !} \sum_{|\mathrm{A}|=q} c^{\mathrm{A}} \otimes e_{\mathrm{A}}=\frac{1}{q !} \sum_{|\mathrm{A}|=q} \sum_{m \in \mathbb{L}} c^{\mathrm{A}}(m) \delta_{m} \otimes e_{\mathrm{A}}
$$

где $c^{\mathrm{A}} \in \mathcal{F}_{0}, c^{\pi \mathrm{A}}=\operatorname{sign} \pi c^{\mathrm{A}}$ для всех $\pi \in \mathfrak{S}_{q}$.

На линейном пространстве $\mathcal{C}_{*}$ линейные отображения $\nabla_{\alpha}, e_{\alpha}^{\wedge}: \mathcal{C}_{*} \rightarrow \mathcal{C}_{*}, \quad \alpha \in[1, D]$, задаются отождествлениями $\nabla_{\alpha} \equiv \nabla_{\alpha} \otimes \mathrm{id}_{\wedge \mathbb{L}}, e_{\alpha}^{\wedge} \equiv \mathrm{id}_{\mathcal{F}_{0}} \otimes e_{\alpha}^{\wedge}$.

Граничный оператор $\partial: \mathcal{C}_{*} \rightarrow \mathcal{C}_{*}$ есть линейное отображение, задаваемое условием

$$
\partial\left(\delta_{m} \otimes e_{\mathrm{A}}\right)=\sum_{1 \leqslant i \leqslant q}(-1)^{i-1}\left(\nabla_{\alpha_{i}} \delta_{m}\right) \otimes e_{\mathrm{A}^{i}}
$$

для всех $m \in \mathbb{L}, \mathrm{A}=\left(\alpha_{1}, \ldots, \alpha_{q}\right), q \in \mathbb{Z}_{+}$и действуюшее по формуле

$$
\mathcal{C}_{q} \ni c=\frac{1}{q !} \sum_{|\mathrm{A}|=q} c^{\mathrm{A}} \otimes e_{\mathrm{A}} \mapsto \partial c=\frac{1}{(q-1) !} \sum_{|\mathrm{A}|=q-1}(\partial c)^{\mathrm{A}} \otimes e_{\mathrm{A}} \in \mathcal{C}_{q-1}
$$

где

$$
(\partial c)^{\mathrm{A}}=\sum_{1 \leqslant \alpha \leqslant D} \nabla_{\alpha} c^{\alpha \mathrm{A}} .
$$

$\mathrm{B}$ частности, $\partial\left(\mathcal{C}_{0}\right)=0$. Для всякой цепи $c \in \mathcal{C}_{*}$ образ $\partial c$ называется ее границей, а сама цепь называется ииклом, если $\partial c=0$.

Равенства $\nabla_{\alpha} \circ \nabla_{\beta}=\nabla_{\beta} \circ \nabla_{\alpha}, \alpha, \beta \in[1, D]$, влекут равенство $\partial \circ \partial=0$, так что определен комплекс $\left\{\mathcal{C}_{*} ; \partial\right\}$ с гомологиями $H_{*}(\mathbb{L})=\bigoplus_{0 \leqslant q<\infty} H_{q}(\mathbb{L})$.

ПРЕДЛОЖЕНИЕ 6.1. Справедливъц равенства

$$
\partial \circ e_{\alpha}^{\wedge}+e_{\alpha}^{\wedge} \circ \partial=\nabla_{\alpha} \quad \partial \Omega я \text { всеx } \quad \alpha \in[1, D]
$$

Доказывается прямыми вычислениями.

6.5. Коцепи на решетке. Линейное пространство коцепей на решетке $\mathbb{L}$ есть прямая сумма $\mathcal{C}^{*} \equiv \mathcal{C}^{*}(\mathbb{L})=\bigoplus_{0 \leqslant q<\infty} \mathcal{C}^{q}$, где линейные пространства $\mathcal{C}^{q}=\left(\mathcal{C}_{q}\right)^{\prime}=$ $\operatorname{Hom}_{\mathbb{C}}\left(\mathcal{C}_{q} ; \mathbb{C}\right)=\mathcal{F} \otimes_{\mathbb{Z}}\left(\wedge^{q} \mathbb{L}^{\prime}\right) \quad\left(\mathcal{C}^{0}=\mathcal{F}, \quad \mathcal{C}^{q}=0\right.$ при $\left.q>D\right)$. Базис в $\mathcal{C}^{q}$ образуют элементарные $q$-коцепи $\delta_{m} \otimes e^{\mathrm{A}}, \quad m \in \mathbb{L}, \mathrm{A}=\left(\alpha_{1}, \ldots, \alpha_{q}\right), \quad 1 \leqslant \alpha_{1}<\cdots<\alpha_{q} \leqslant D$, так что каждая $q$-коцеп $\omega \in \mathcal{C}^{q}$ имеет однозначное представление

$$
\omega=\frac{1}{q !} \sum_{|\mathrm{A}|=q} \omega_{\mathrm{A}} \otimes e^{\mathrm{A}}=\frac{1}{q !} \sum_{|\mathrm{A}|=q} \sum_{m \in \mathbb{L}} \omega_{\mathrm{A}}(m) \delta_{m} \otimes e^{\mathrm{A}}
$$


где $\omega_{\mathrm{A}} \in \mathcal{F}, \omega_{\pi \mathrm{A}}=\operatorname{sign} \pi \omega_{\mathrm{A}}$ для всех $\pi \in \mathfrak{S}_{q}$. Спаривание $\langle\omega, c\rangle \in \mathbb{C}$ элементов

$$
c=\frac{1}{q !} \sum_{|\mathrm{A}|=q} c^{\mathrm{A}} \otimes e_{\mathrm{A}} \in \mathcal{C}_{q}, \quad \omega=\frac{1}{q !} \sum_{|\mathrm{A}|=q} \omega_{\mathrm{A}} \otimes e^{\mathrm{A}} \in \mathcal{C}^{q}, \quad q \in \mathbb{Z}_{+},
$$

вычисляется по формуле

$$
\langle\omega, c\rangle=\frac{1}{q !} \sum_{|\mathrm{A}|=q}\left\langle\omega_{\mathrm{A}}, c^{\mathrm{A}}\right\rangle
$$

$\mathrm{B}$ частности, $\omega_{\mathrm{A}}(m)=\left\langle\omega, \delta_{m} \otimes e_{\mathrm{A}}\right\rangle$ для

$$
\omega=\frac{1}{q !} \sum_{|\mathrm{A}|=q} \sum_{m \in \mathbb{L}} \omega_{\mathrm{A}}(m) \delta_{m} \otimes e^{\mathrm{A}} .
$$

Линейные отображения $\Delta_{\alpha}, \iota_{\alpha}, d_{\mathrm{R}}: \mathcal{C}^{*} \rightarrow \mathcal{C}^{*}, \alpha \in[1, D]$, дуальные к отображениям $\nabla_{\alpha}, e_{\alpha}^{\wedge}, \partial: \mathcal{C}_{*} \rightarrow \mathcal{C}_{*}$, задаются отождествлениями $\Delta_{\alpha} \equiv \Delta_{\alpha} \otimes \operatorname{id}_{\wedge \mathbb{L}^{\prime}}, \iota_{\alpha} \equiv \operatorname{id}_{\mathcal{F}} \otimes \iota_{\alpha}$ и формулой

$$
\mathcal{C}^{q} \ni \omega=\frac{1}{q !} \sum_{|\mathrm{A}|=q} \omega_{\mathrm{A}} \otimes e^{\mathrm{A}} \mapsto d_{\mathrm{R}} \omega=\frac{1}{(q+1) !} \sum_{|\mathrm{A}|=q+1}\left(d_{\mathrm{R}} \omega\right)_{\mathrm{A}} \otimes e^{\mathrm{A}} \in \mathcal{C}^{q+1},
$$

где

$$
\left(d_{\mathrm{R}} \omega\right)_{\mathrm{A}}=\sum_{0 \leqslant i \leqslant q}(-1)^{i} \Delta_{\alpha_{i}} \omega_{\mathrm{A}^{i}}, \quad \mathrm{~A}=\left(\alpha_{0}, \ldots, \alpha_{q}\right) .
$$

Для всякой коцепи $\omega \in \mathcal{C}^{*}$ образ $d_{\mathrm{R}} \omega$ называется ее кограницей, а сама коцепь называется коииклом, если $d_{\mathrm{R}} \omega=0$. Равенство $\partial \circ \partial=0$ влечет $d_{\mathrm{R}} \circ d_{\mathrm{R}}=0$, так что определен комплекс $\left\{\mathcal{C}^{*} ; d_{\mathrm{R}}\right\}$ с когомологиями $H^{*}(\mathbb{L})=\bigoplus_{0 \leqslant q<\infty} H^{q}(\mathbb{L})$. Этот комплекс есть дискретный аналог комплекса де Рама гладкого многообразия, индекс R у дифференциала $d_{\mathrm{R}}$ подчеркивает этот факт и отличает его от дифференциала $d$ в основном тексте. Предложение 6.1 имеет дуальное

ПРЕДЛОЖЕНИЕ 6.2. Справедливъ равенства

$$
d_{\mathrm{R}} \circ \iota_{\alpha}+\iota_{\alpha} \circ d_{\mathrm{R}}=\Delta_{\alpha} \quad \text { для всех } \quad \alpha \in[1, D] .
$$

6.6. Гомотопическая формула для коцепей. Для всякой точки

$$
m=\left(\mu^{1}, \ldots, \mu^{D}\right)=\sum_{1 \leqslant \alpha \leqslant D} \mu^{\alpha} e_{\alpha} \in \mathbb{L}
$$

положим

$$
m_{(0)}=0, \quad m_{(\alpha)}=\sum_{1 \leqslant \beta \leqslant \alpha} \mu^{\beta} e_{\beta}, \quad \alpha \in[1, D],
$$

так что $m_{(D)}=m$. Линейные отображения $S^{\alpha}: \mathcal{F} \rightarrow \mathcal{F}, \alpha \in[1, D]$, зададим формулами

$$
S^{\alpha} f(m)= \begin{cases}\sum_{0 \leqslant \mu<\mu^{\alpha}} f\left(m_{(\alpha-1)}+\mu e_{\alpha}\right), & \mu^{\alpha}>0, \\ 0, & \mu^{\alpha}=0, \\ -\sum_{\mu^{\alpha} \leqslant \mu<0} f\left(m_{(\alpha-1)}+\mu e_{\alpha}\right), & \mu^{\alpha}<0,\end{cases}
$$

для всех $f \in \mathcal{F}$. (Эти формулы - дискретный аналог интегрирования по направленному отрезку $\left[m_{(\alpha-1)}, m_{(\alpha)}\right]$; заметим, что $S^{\alpha}\left(\mathcal{F}_{0}\right) \nsubseteq \mathcal{F}_{0}$.) 
ПреДЛОЖЕнИЕ 6.3. Пусть $f \in \mathcal{F}$, тогда

1) для всех $\alpha, \beta \in[1, D]$ имеем

$$
\Delta_{\alpha} S^{\beta} f(m)=\left\{\begin{array}{cc}
S^{\beta} \Delta_{\alpha} f(m), & \alpha<\beta \\
f\left(m_{(\beta)}\right), & \alpha=\beta \\
0, & \alpha>\beta
\end{array}\right.
$$

2) для всех $\alpha \in[1, D]$ имеем

$$
S^{\alpha} \Delta_{\alpha} f(m)=\left.f\right|_{m_{(\alpha-1)}} ^{m_{(\alpha)}}=f\left(m_{(\alpha)}\right)-f\left(m_{(\alpha-1)}\right) .
$$

Доказывается прямыми вычислениями.

Определим линейное отображение $\mathfrak{h}: \mathcal{C}^{*} \rightarrow \mathcal{C}^{*}$ правилами

$$
\begin{aligned}
\mathcal{C}^{0} & =\mathcal{F} \ni f \mapsto \mathfrak{h} f=0, \\
\mathcal{C}^{q} \ni \omega=\frac{1}{q !} \sum_{|\mathrm{A}|=q} \omega_{\mathrm{A}} \otimes e^{\mathrm{A}} & \mapsto \mathfrak{h} \omega=\frac{1}{(q-1) !} \sum_{|\mathrm{A}|=q-1}(\mathfrak{h} \omega)_{\mathrm{A}} \otimes e^{\mathrm{A}} \in \mathcal{C}^{q-1},
\end{aligned}
$$

где

$$
(\mathfrak{h} \omega)_{\mathrm{A}}(m)=\sum_{\alpha>\mathrm{A}}\left(S^{\alpha} \omega_{\alpha \mathrm{A}}\right)(m)
$$

для всех $\omega \in \mathcal{C}^{q}, \mathrm{~A}=\left(\alpha_{1}, \ldots, \alpha_{q-1}\right), m \in \mathbb{L}, q \in[1, D]$, запись $\alpha>\mathrm{A}$ означает, что $\alpha>\alpha_{i}$ для всех $i \in[1, q-1]$. Линейное отображение $\rho: \mathcal{C}^{*} \rightarrow \mathcal{C}^{*}$ определим правилом $\mathcal{C}^{0} \ni f \mapsto \rho f \in \mathcal{C}^{0}, \rho f(m)=f(0)$ для всех $m \in \mathbb{L}$ и $\rho \omega=0$ для всех $\omega \in \mathcal{C}^{q}, q \in[1, D]$.

Лемма 6.1. Справедлива гомотопическая формула

$$
d_{\mathrm{R}} \circ \mathfrak{h}+\mathfrak{h} \circ d_{\mathrm{R}}=\mathrm{id}-\rho .
$$

ДОКАЗАТЕЛЬСТвО. ПУсть

$$
q \in[1, D], \quad \omega=\frac{1}{q !} \sum_{|\mathrm{A}|=q} \omega_{\mathrm{A}} \otimes e^{\mathrm{A}} \in \mathcal{C}^{q}, \quad \mathrm{~A}=\left(\alpha_{1}, \ldots, \alpha_{q}\right) .
$$

Тогда

$$
\begin{aligned}
\left(d_{\mathrm{R}} \mathfrak{h} \omega\right)_{\mathrm{A}}(m)= & \sum_{1 \leqslant i \leqslant q}(-1)^{i-1} \Delta_{\alpha_{i}}(\mathfrak{h} \omega)_{\mathrm{A}^{i}}(m)= \\
= & \sum_{1 \leqslant i \leqslant q}(-1)^{i-1} \Delta_{\alpha_{i}} \sum_{\beta>\mathrm{A}^{i}}\left(S^{\beta} \omega_{\beta \mathrm{A}^{i}}\right)(m)= \\
= & (-1)^{i_{\max }-1} \Delta_{\alpha_{\max }} \sum_{\beta \geqslant \alpha_{\max }}\left(S^{\beta} \omega_{\beta \mathrm{A}^{i \max }}\right)(m)+ \\
& +\sum_{i \neq i_{\max }}(-1)^{i-1} \Delta_{\alpha_{i}} \sum_{\beta>\alpha_{\max }}\left(S^{\beta} \omega_{\beta \mathrm{A}^{i}}\right)(m)= \\
= & \omega_{\mathrm{A}}\left(m_{\left(\alpha_{\max }\right)}\right)+\sum_{1 \leqslant i \leqslant q}(-1)^{i-1} \sum_{\beta>\alpha_{\max }}\left(S^{\beta} \Delta_{\alpha_{i}} \omega_{\beta \mathrm{A}^{i}}\right)(m),
\end{aligned}
$$


где $\alpha_{\max } \equiv \alpha_{i_{\max }}>\alpha_{i}$ для всех $i \in[1, q], i \neq i_{\max }$, и мы воспользовались предложением 6.3(1). С другой стороны,

$$
\begin{aligned}
\left(\mathfrak{h} d_{\mathrm{R}} \omega\right)_{\mathrm{A}}(m) & =\sum_{\beta>\mathrm{A}}\left(S^{\beta}\left(d_{\mathrm{R}} \omega\right)_{\beta \mathrm{A}}\right)(m)= \\
& =\sum_{\beta>\alpha_{\max }} S^{\beta}\left(\Delta_{\beta} \omega_{\mathrm{A}}+\sum_{1 \leqslant i \leqslant q}(-1)^{i} \Delta_{\alpha_{i}} \omega_{\beta \mathrm{A}^{i}}\right)(m)= \\
& =\left.\omega_{\mathrm{A}}\right|_{m_{\left(\alpha_{\max }\right)}^{m}}+\sum_{\beta>\alpha_{\max }} \sum_{1 \leqslant i \leqslant q}(-1)^{i}\left(S^{\beta} \Delta_{\alpha_{i}} \omega_{\beta \mathrm{A}^{i}}\right)(m),
\end{aligned}
$$

где мы воспользовались предложением 6.3(2) и учли, что $m_{(D)}=m$. Складывая полученные представления, убеждаемся в справедливости гомотопической формулы для $q \in[1, D]$. Для $q=0$ проверка элементарна.

Теорема 6.1. Справедливы равенства

$$
H^{q}(\mathbb{L})= \begin{cases}\mathbb{C}, & q=0 \\ 0, & q>0\end{cases}
$$

ДокАЗАТЕЛЬСТво. Достаточно воспользоваться доказанной гомотопической формулой.

6.7. Гомотопическая формула для цепей. Введенное выше линейное отображение $\mathfrak{h}: \mathcal{C}^{*} \rightarrow \mathcal{C}^{*}$ имеет дуальное отображение $\mathfrak{g}: \mathcal{C}_{*} \rightarrow \mathcal{C}_{*}$, задаваемое условием $\langle\omega, \mathfrak{g} c\rangle=$ $\langle\mathfrak{h} \omega, c\rangle$ для всех $c \in \mathcal{C}_{q}, \omega \in \mathcal{C}^{q+1}$. Явный вид дуального отображения достаточно громоздкий и нам не важен. Важно лишь, что гомотопическая формула для коцепей из леммы 6.1 приводит к дуальной формуле для цепей

$$
\mathfrak{g} \circ \partial+\partial \circ \mathfrak{g}=\mathrm{id}-\phi .
$$

Здесь линейное отображение $\phi: \mathcal{C}_{q} \rightarrow \mathcal{C}_{q}$, дуальное к отображению $\rho$, задается условием $\langle\phi c, \omega\rangle=\langle c, \rho \omega\rangle$ для всех $c \in \mathcal{C}_{q}, \omega \in \mathcal{C}^{q}, q \in[0, D]$ и действует по правилу $\phi g=(\Sigma g) \delta$ для $g \in \mathcal{C}_{0}=\mathcal{F}_{0}, \phi c=0$ для всех $c \in \mathcal{C}_{q}, q \in[1, D]$, где $\Sigma g=\sum_{m \in \mathbb{L}} g(m) \in \mathbb{C}, \delta(m)=1$ при $m=0$ и $\delta(m)=0$ при $m \neq 0$ (т.е. $\delta=\delta_{0} \in \mathcal{F}_{0}$ ). В частности,

$$
H_{q}(\mathbb{L})= \begin{cases}\mathbb{C}, & q=0 \\ 0, & q>0\end{cases}
$$

6.8. Связь с когомологиями Шевалле-Эйленберга. Линейное пространство $\mathbb{V}=\mathbb{C} \otimes_{\mathbb{Z}} \mathbb{L}=\mathbb{C}^{D}$ имеет базис $e_{1}, \ldots, e_{D} \in \mathbb{L}$, так что каждый элемент $u \in \mathbb{V}$ однозначно записывается в виде

$$
u=\left(u^{1}, \ldots, u^{D}\right)=\sum_{0 \leqslant \alpha \leqslant D} u^{\alpha} e_{\mathrm{A}},
$$

где $u^{\alpha} \in \mathbb{C}, \alpha \in[1, D]$. Определим на $\mathbb{V}$ структуру коммутативной алгебры Ли, положив $[u, v]=0$ для всех $u, v \in \mathbb{V}$, и зададим представление этой алгебры Ли в линейном 
пространстве $\mathcal{F}$ правилом $\mathbb{V} \ni e_{\alpha} \mapsto \Delta_{\alpha} \in \mathfrak{g l}(\mathcal{F})$ для всех $\alpha \in[1, D]$, где $\mathfrak{g l}(\mathcal{F})$ - алгебра Ли эндоморфизмов линейного пространства $\mathcal{F}$ с коммутатором в качестве скобки Ли.

Линейное пространство кочепей на алгебре Ли $\mathbb{V}$ с коэффициентами в линейном пространстве $\mathcal{F}$ есть $\mathcal{C}_{\mathrm{CE}}^{*}(\mathbb{V} ; \mathcal{F})=\bigoplus_{0 \leqslant q<\infty} \mathcal{C}_{\mathrm{CE}}^{q}(\mathbb{V} ; \mathcal{F})$, где $\mathcal{C}_{\mathrm{CE}}^{q}(\mathbb{V} ; \mathcal{F})=$ $\operatorname{Hom}_{\mathbb{C}}\left(\wedge^{q} \mathbb{V} ; \mathcal{F}\right)$ суть линейные пространства всех $q$-линейных кососимметрических отображений из $\mathbb{V}^{q}=\times^{q} \mathbb{V}$ в $\mathcal{F}$. (Заметим, что $\mathcal{C}_{\mathrm{CE}}^{0}(\mathbb{V} ; \mathcal{F})=\mathcal{F}, \mathcal{C}_{\mathrm{CE}}^{q}(\mathbb{V} ; \mathcal{F})=0$ при $q>D$.) Внешний дифференциал $d_{\mathrm{CE}}: \mathcal{C}_{\mathrm{CE}}^{*}(\mathbb{V} ; \mathcal{F}) \rightarrow \mathcal{C}_{\mathrm{CE}}^{*}(\mathbb{V} ; \mathcal{F})$ задается формулой

$$
d_{\mathrm{CE}} \chi\left(e_{\alpha_{0}} \wedge \cdots \wedge e_{\alpha_{q}}\right)=\sum_{0 \leqslant i \leqslant q}(-1)^{i} \Delta_{\alpha_{i}} \chi\left(e_{\alpha_{0}} \wedge \ldots e_{\alpha_{i}}^{\check{1}} \cdots \wedge e_{\alpha_{q}}\right)
$$

для всех $\chi \in \mathcal{C}_{\mathrm{CE}}^{q}(\mathbb{V} ; \mathcal{F}), \alpha_{0}, \ldots, \alpha_{q} \in[1, D], q \in[0, D]$. Легко проверяется, что $d_{\mathrm{CE}}$ ○ $d_{\mathrm{CE}}=0$, так что определен комплекс $\left\{\mathcal{C}_{\mathrm{CE}}^{*}(\mathbb{V} ; \mathcal{F}) ; d_{\mathrm{CE}}\right\}$ с когомологиями $H_{\mathrm{CE}}^{*}(\mathbb{V} ; \mathcal{F})=$ $\bigoplus_{0 \leqslant q<\infty} H_{\mathrm{CE}}^{q}(\mathbb{V} ; \mathcal{F})$.

ПРЕДЛОЖЕНИЕ 6.4. Правило

$$
\mathcal{C}^{q} \ni \omega=\frac{1}{q !} \sum_{|\mathrm{A}|=q} \omega_{\mathrm{A}} \otimes e^{\mathrm{A}} \mapsto \chi \in \mathcal{C}_{\mathrm{CE}}^{q}(\mathbb{V} ; \mathcal{F}), \quad q \in[0, D]
$$

где $\chi: \wedge^{q} \mathbb{V} \rightarrow \mathcal{F}$ по формуле $\chi\left(e_{\alpha_{1}} \wedge \cdots \wedge e_{\alpha_{q}}\right)=\omega_{\alpha_{1} \ldots \alpha_{q}}$ для всех $\alpha_{1}, \ldots, \alpha_{q} \in[1, D]$, задает изоморфизм комплексов

$$
\left\{\mathcal{C}^{*} ; d_{\mathrm{R}}\right\} \simeq\left\{\mathcal{C}_{\mathrm{CE}}^{*}(\mathbb{V} ; \mathcal{F}) ; d_{\mathrm{CE}}\right\}
$$

и соответствующих когомологий.

ДОКАЗАТЕЛЬСТВО непосредственно следует из определений.

Благодарности. Работа выполнена при поддержке грантов РФФИ № 04-01-00769 и НШ-1542.2003.1. Я благодарен Ю. М. Зиновьеву за полезное обсуждение когомологий де Рама на решетке, а также за оттиск статьи [7].

\section{Список литературы}

[1] В. В. Жаринов. ТМФ. 2004. Т. 140. № 3. С. 355-366.

[2] В. В. Жаринов. ТМФ. 2001. Т. 128. № 2. С. 147-160.

[3] В. В. Жаринов. ТМФ. 2003. Т. 136. № 2. С. 179-196.

[4] J. L. Taylor. Adv. Math. 1972. V. 9. P. 137-182.

[5] В. С. Владимиров. Обобщенные функции в математической физике. М.: Наука, 1979.

[6] A. А. Дезин. Многомерный анализ и дискретные модели. М.: Наука, 1990.

[7] Yu. M. Zinoviev. Commun. Math. Phys. 1995. V. 168. P. 227-247.

Поступила в редакцию 11.I.2005 г. 